\title{
Reliability Analysis of Key Components of the Pod Based on Grey System Theory
}

\author{
Dongfang Hu $\mathbb{D}^{1,2}$ Hang Su $\mathbb{D}^{1,2}$ and Zuhui Shen ${ }^{3}$ \\ ${ }^{1}$ School of Mechatronics Engineering, Henan University of Science and Technology, Luoyang 471003, China \\ ${ }^{2}$ Collaborative Innovation Center of Machinery Equipment Advanced Manufacturing of Henan Province, \\ Luoyang 471003, China \\ ${ }^{3}$ Xinxiang Vocational and Technical College, Xinxiang 453000, China \\ Correspondence should be addressed to Hang Su; suhang509@163.com
}

Received 22 June 2020; Accepted 30 July 2020; Published 17 August 2020

Academic Editor: Alessandro Gasparetto

Copyright ( $\odot 2020$ Dongfang Hu et al. This is an open access article distributed under the Creative Commons Attribution License, which permits unrestricted use, distribution, and reproduction in any medium, provided the original work is properly cited.

\begin{abstract}
For improving the reliability of key components in an airborne pod, gray system theory is introduced into the virtual design of airborne pod. Based on this theory, key components of airborne pod are analyzed and mathematical models of the gray relational model and absolute and relative gray relational model are established, respectively. The differences and relations of the three methods for evaluating the robustness of products are researched. Considering the influence of relevant factors, the change rate of each evaluation index is calculated, and the reliability analysis of the airborne pod body is realized ultimately. Finally, the No. 2 test pod body is the optimal solution, which provides an effective theoretical basis for determining the optimal structure of the airborne pod body.
\end{abstract}

\section{Introduction}

For any mechanical system, in order to make the design, development, and manufacturing reliable, that is, to study its reliability, it is necessary to study the reliability of a mechanical system. Mechanical systems are composed of many factors. For the grey system [1], the reliability of a mechanical engineering system describes the ability of the system to accurately, timely, and coordinately complete specified tasks under specified conditions and within a specified time. When this ability is measured with probability, it is reliability [2]. In the service process of a mechanical system, a reliable mechanical system will play a vital role in guaranteeing its working performance.

In recent years, scholars have conducted a series of research and exploration in the field of reliability analysis in view of the diversity and complexity of mechanical structure reliability. The most commonly used methods are probability statistics, fuzzy mathematics, evidence theory, and gray system theory. After consulting the relevant literature, the application research of uncertain systems in various engineering fields has made corresponding progress, for example, combining the analysis method and the checkpoint method with the MATLAB software platform to improve the reliability of key component analysis [3]. The gray system is extended to the theory of probability statistics, the gray probability density function and gray reliability calculation method are studied, and the gray reliability calculation model of stress intensity interference is established [4]. A generalized SPE flux sequence and an extreme value SPE flux sequence taking the occurrence time as a sequence are proposed, and a solar proton event prediction method based on gray GM $(1,1)$ and interval is established [5]. An algorithm based on the self-help method and fuzzy mathematics is proposed, and a fuzzy self-help fuzzy mathematics model based on fusion of bad information objects is established. The measured data of bad information characteristic information objects are self-sampled, and the maximum entropy algorithm is used to obtain the measured data. Selfservice distribution of data: self-service fusion sequence uses self-service distribution to estimate the true value and interval of the measured pressure value through the fuzzy 
membership function [6]. Combining the evidence theory analysis method, an effective model and an efficient and reliable mechanical structure are proposed [7].

However, according to the relevant literature review, the existing research content rarely sees the grayness in the structural design of aeronautical products, especially airborne equipment. This article is based on this purpose.

\section{Establishment of the Mathematical Model}

2.1. Deng's Grey System Theory. If the behavior factor of a certain aspect of the mechanical system is $X_{0}$ and $X_{0}$ is affected by multiple factors $x_{i}(i=1,2, \ldots, n)$, then this method of using the gray correlation degree of factor $x_{i}$ to factor $X_{0}$ to express the influence of $x_{i}$ on $X_{0}$ is called gray correlation analysis. The method in which $X_{0}$ affects the size is called the gray relational analysis method. Based on Deng's gray relational analysis theory, the reliability of the outer frame of the airborne pod is regarded as a gray system, and its structural reliability assessment is determined by various performance indicators.

Selecting the structural reliability of the pod cabin as the research object and introducing the concept of behavior factors, the cabin structure evaluation index (one of the reliability evaluation indicators described in the technical parameter file of the airborne pod) $X_{0}$ system characteristic behavior sequence is

$$
X_{0}=\left\{x_{0}(1), x_{0}(2), \ldots, x_{0}(k), \ldots, x_{0}(n)\right\} .
$$

In the formula, it is the first evaluation data of a certain behavioral factor.

According to the relevant technical requirements of airborne pod, the reliability of the pod structure depends on the interaction of multiple behavior factors. Based on this, a gray relational mathematical model is constructed.

Theorem 1. Assume that there are all kinds of factors affecting the reliability of products. By testing and extracting relevant data, the sequence of behavioral indicators of the first $(i \leq m)$ factor is obtained as follows:

$$
X_{i}=\left\{x_{i}(1), x_{i}(2), \ldots, x_{i}(k), \ldots, x_{i}(n)\right\} .
$$

In the formula, $I$ represents the number of the first influencing factors, $i=1,2, \ldots, m ; K$ is the serial number of data, $k=1,2, \ldots, n$; and $x_{i}(k)$ is the $K$ measured value of influencing factor $x_{i}$.

From formulas (1) and (2), the corresponding forms of the sequence of system characteristic behavior $X_{0}$ and $X_{i}$ comparison data are deduced, which are, in turn, as follows:

$$
\begin{aligned}
X_{0} & =\left\{x_{0}(1), x_{0}(2), \ldots, x_{0}(k), \ldots, x_{0}(n)\right\}, \\
X_{i} & =\left\{x_{i}(1), x_{i}(2), \ldots, x_{i}(k), \ldots, x_{i}(n)\right\}, \\
X_{m} & =\left\{x_{m}(1), x_{m}(2), \ldots, x_{m}(k), \ldots, x_{m}(n)\right\} .
\end{aligned}
$$

\subsection{Determination of Mechanical Reliability Parameters.} Mechanical reliability can generally be divided into structural reliability and mechanical reliability. Structural reliability mainly considers the strength of the mechanical structure and the failure caused by fatigue, wear, and fracture under load; the mechanism reliability does not consider the failure caused by the strength, but the failure caused by the kinematics during the action, so the reliability is not bias.

As showed in Figure 1, the appearance of the pod body frame is generally spherical. According to the design technical parameters of the pod body, such as the azimuth attitude during flight, the symmetrical shape of aerodynamic characteristics, the extreme environmental requirements at high and low temperatures, the air tightness requirements of the internal environment, the antivibration, and anti-impact requirements, 30 closely related evaluation indicators are determined.

Due to the difference of units between data, there are obvious differences in the order of magnitude of each other. It is obviously necessary to integrate data in dimension. Referring to the evaluation criteria of aeronautical product quality parameters, it is artificially divided into 10 grades: when the evaluation coefficient is $8-10$, the index is good, when the evaluation coefficient is 6-7, the index is general, and when the index is $4-5$, the index is secondary. Based on 30 performance indicators, the performance of the virtual prototype was tested sequentially. According to the test data, 15 indicators for an evaluation coefficient of 6 were selected as the key factors. According to the given technical requirements document for pod design, the 15 evaluation indexes are sorted from important to general according to the key parts embodied in the design document, as shown in Table 1.

According to the research emphasis, the structural reliability of the pod body is selected as the research object, and the system characteristic behavior sequence composed of 15 evaluation indexes of the airborne pod body is obtained:

$$
X_{0}=\{7,9,6,8,7,7,8,6,8,8,9,7,6,7,8\} .
$$

In the simulation environment, 10 sets of representative virtual test prototypes are selected from a large number of random test prototypes (i.e., random samples) according to different design ideas. According to the abovementioned performance tests, the evaluation tests of the abovementioned 15 indicators were carried out, respectively. According to the corresponding experimental conditions, 10 sets of comparative data sequences of different results are obtained: 


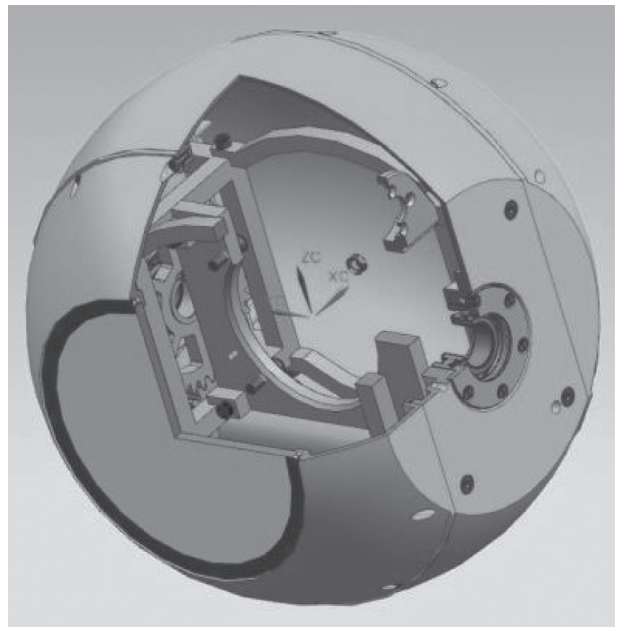

FIGURE 1: Solid modeling of the pod frame.

TABle 1: Assessment sequence of reliability parameters for airborne pods.

\begin{tabular}{lccc}
\hline $\begin{array}{l}\text { Serial } \\
\text { number }\end{array}$ & Symbol $t$ & Echnical parameter & Evaluation coefficient ( $\leq 10)$ \\
\hline 1 & $x_{0}(1)$ & Strength and stiffness of outer spherical shells & 7 (good) \\
2 & $x_{0}(2)$ & Strength and stiffness of the inner frame & 9 (perfect) \\
3 & $x_{0}(3)$ & Antivibration characteristics of outer spherical shells & 6 (good) \\
4 & $x_{0}(4)$ & Vibration resistance of the inner frame & 8 (perfect) \\
5 & $x_{0}(5)$ & Symmetry characteristics of outer spherical shells & 7 (good) \\
6 & $x_{0}(6)$ & Symmetry characteristic of the inner frame & 7 (good) \\
7 & $x_{0}(7)$ & Gas tightness characteristics of the outer spherical shell & 8 (perfect) \\
8 & $x_{0}(8)$ & Corrosion resistance of outer spherical shells & 6 (good) \\
9 & $x_{0}(9)$ & Corrosion resistance of the inner frame & 8 (perfect) \\
10 & $x_{0}(10)$ & Temperature characteristics of outer spherical shell materials & 8 (perfect) \\
11 & $x_{0}(11)$ & Temperature characteristics of inner frame materials & 9 (perfect) \\
12 & $x_{0}(12)$ & Vibration resistance of the azimuth motor & 7 (good) \\
13 & $x_{0}(13)$ & Strength and stiffness of connectors & 6 (good) \\
14 & $x_{0}(14)$ & Fatigue resistance of the bearing drive & 7 (good) \\
15 & $x_{0}(15)$ & Impact resistance of the bearing drive & 8 (perfect) \\
\hline
\end{tabular}

$$
\begin{aligned}
& X_{1}=\{7.7,9.1,6.5,8.3,7.4,7.7,8.6,6.2,8.6,8.4,9.3,7.9,6.1,7.4,8.5\}, \\
& X_{2}=\{7.3,9.4,6.1,8.7,7.6,7.3,8.4,6.5,8.3,8.1,9.5,7.5,6.2,7.1,8.6\}, \\
& X_{3}=\{7.1,9.3,6.7,8.4,7.3,7.1,8.2,6.8,8.1,8.8,9.3,7.3,6.7,7.3,8.7\}, \\
& X_{4}=\{7.6,9.2,6.4,8.7,7.2,7.7,8.6,6.1,8.2,8.7,9.1,7.9,6.5,7.4,8.2\}, \\
& X_{5}=\{7.8,9.1,6.2,8.6,7.7,7.3,8.4,6.4,8.6,8.1,9.5,7.4,6.1,7.3,8.1\}, \\
& X_{6}=\{7.3,9.5,6.6,8.1,7.3,7.6,8.1,6.7,8.9,8.4,9.3,7.2,6.5,7.4,8.6\}, \\
& X_{7}=\{7.7,9.2,6.3,8.5,7.7,7.3,8.5,6.3,8.2,8.6,9.4,7.5,6.1,7.3,8.4\}, \\
& X_{8}=\{7.2,9.5,6.6,8.2,7.4,7.8,8.2,6.6,8.7,8.5,9.2,7.3,6.5,7.1,8.7\}, \\
& X_{9}=\{7.5,9.3,6.8,8.3,7.6,7.1,8.6,6.1,8.3,8.7,9.1,7.1,6.8,7.3,8.3\}, \\
& X_{10}=\{7.8,9.2,6.1,8.5,7.3,7.8,8.4,6.9,8.7,8.1,9.3,7.5,6.1,7.7,8.6\}
\end{aligned}
$$


Thus, a preliminary data sample for evaluating the reliability of the pod structure is obtained.

\section{Establishment of Dun's Grey Correlation Model}

3.1. Interval Value Processing. Based on the correlation degree problem in the gray system theory, it is known that the reliability analysis is mainly to characterize the similarity degree of the system characteristic behavior line graphs of each comparison data line, and the representation index is exactly 15 points, selected according to the point coordinates. The similarity on the graph is used to estimate the result. Then, the evaluation range $1 \sim 10$ will be the key to the interval value, and the interval value method will be selected for processing. The interval-valued operator will be introduced to obtain the following relationship:

$$
\begin{aligned}
X_{i} & =\left\{x_{i}(1), x_{i}(2), \ldots, x_{i}(k), \ldots, x_{i}(n)\right\}, \\
X_{i} D & =\left\{x_{i}(1) d, x_{i}(2) d, \ldots, x_{i}(k) d, \ldots, x_{i}(n) d\right\}, \\
x_{i}(k) d & =\frac{x_{i}(k)-\min x_{i}(k)}{\max x_{i}(k)-\min x_{i}(k)}, \quad k=1,2, \ldots, n .
\end{aligned}
$$

Then, according to the interval operator quoted by equations (6) and (7), 11 sets of different data $\left(X_{0}\right.$ is the feature sequence) $X_{0}, X_{1}, X_{2}, \ldots, X_{10}$ are interval-valued, and the gray system algorithm program is written by Microsoft Visual $\mathrm{C}++$ software. The interval-valued data sequence is

$$
\begin{aligned}
& X_{0}^{\prime}=\{0.33,1.00,0.00,0.67,0.33,0.33,0.67,0.00,0.67,0.67,1.00,0.33,0.00,0.33,0.67\}, \\
& X_{1}^{\prime}=\{0.50,0.94,0.13,0.69,0.41,0.50,0.78,0.03,0.78,0.72,1.00,0.56,0.00,0.41,0.75\}, \\
& X_{2}^{\prime}=\{0.35,0.97,0.00,0.76,0.44,0.35,0.68,0.12,0.65,0.59,1.00,0.41,0.03,0.29,0.74\}, \\
& X_{3}^{\prime}=\{0.15,1.00,0.00,0.65,0.23,0.15,0.58,0.04,0.54,0.81,1.00,0.23,0.00,0.23,0.77\}, \\
& X_{4}^{\prime}=\{0.48,1.00,0.10,0.84,0.35,0.52,0.81,0.00,0.68,0.84,0.97,0.58,0.13,0.42,0.68\}, \\
& X_{5}^{\prime}=\{0.50,0.88,0.03,0.74,0.47,0.35,0.68,0.09,0.74,0.59,1.00,0.38,0.00,0.35,0.59\}, \\
& X_{6}^{\prime}=\{0.27,1.00,0.03,0.53,0.27,0.37,0.53,0.07,0.80,0.63,0.93,0.23,0.00,0.30,0.70\}, \\
& X_{7}^{\prime}=\{0.48,0.94,0.06,0.73,0.48,0.36,0.73,0.06,0.64,0.76,1.00,0.42,0.00,0.36,0.70\}, \\
& X_{8}^{\prime}=\{0.23,1.00,0.03,0.57,0.30,0.43,0.57,0.03,0.73,0.67,0.90,0.27,0.00,0.20,0.73\}, \\
& X_{9}^{\prime}=\{0.44,1.00,0.22,0.69,0.47,0.31,0.78,0.00,0.69,0.81,0.94,0.31,0.22,0.38,0.69\}, \\
& X_{10}^{\prime}=\{0.53,0.97,0.00,0.75,0.38,0.53,0.72,0.25,0.81,0.63,1.00,0.44,0.00,0.50,0.78\}
\end{aligned}
$$

3.2. Difference Sequence and Range. The 11 sets of new sequences derived after interval-valued are, respectively, recorded as $X_{0}^{\prime}, X_{1}^{\prime}, X_{2}^{\prime}, \ldots, X_{10}^{\prime}$, and the following relations are derived from the four axioms of gray correlation [8]:

$$
\begin{aligned}
\Delta_{i}(k) & =\left|x_{0}^{\prime}(k)-x_{i}^{\prime}(k)\right|, \\
\Delta_{i} & =\left(\Delta_{i}(1), \Delta_{i}(2), \ldots, \Delta_{i}(n)\right), \quad i=0,1,2, \ldots, m .
\end{aligned}
$$

According to equations (9) and (10), the difference data sequence (retaining 2 significant digits) is obtained, which essentially calculates the geometric distance between the corresponding two points:

$$
\begin{aligned}
& \Delta_{1}=\{0.17,0.06,0.13,0.02,0.08,0.17,0.11,0.03,0.11,0.05,0.00,0.23,0.00,0.08,0.08\}, \\
& \Delta_{2}=\{0.02,0.03,0.00,0.09,0.11,0.02,0.01,0.12,0.02,0.08,0.00,0.08,0.03,0.04,0.07\}, \\
& \Delta_{3}=\{0.18,0.00,0.00,0.02,0.10,0.18,0.09,0.04,0.13,0.14,0.00,0.10,0.00,0.10,0.10\}, \\
& \Delta_{4}=\{0.15,0.00,0.10,0.17,0.02,0.19,0.14,0.00,0.01,0.17,0.03,0.25,0.13,0.09,0.01\}, \\
& \Delta_{5}=\{0.17,0.12,0.03,0.07,0.14,0.02,0.01,0.09,0.07,0.08,0.00,0.05,0.00,0.02,0.08\}, \\
& \Delta_{6}=\{0.06,0.00,0.03,0.14,0.06,0.04,0.14,0.07,0.13,0.04,0.07,0.10,0.00,0.03,0.03\}, \\
& \Delta_{7}=\{0.15,0.06,0.06,0.06,0.15,0.03,0.06,0.06,0.03,0.09,0.00,0.09,0.00,0.03,0.03\}, \\
& \Delta_{8}=\{0.10,0.00,0.03,0.10,0.03,0.10,0.10,0.03,0.06 .0 .06,0.10,0.06,0.00,0.13,0.06\}, \\
& \Delta_{9}=\{0.11,0.00,0.22,0.02,0.14,0.02,0.11,0.00,0.02,0.14,0.06,0.02,0.22,0.05,0.02\}, \\
& \Delta_{10}=\{0.20,0.03,0.00,0.08,0.05,0.20,0.05,0.25,0.14,0.04,0.00,0.11,0.00,0.17,0.11\} .
\end{aligned}
$$


For each set of different sequences, the data are retrieved according to the written $\mathrm{C}++$ program, and the maximum and minimum values are, respectively, recorded as

$$
\begin{gathered}
M=\max _{i} \max _{k} \Delta_{i}(k), \\
m=\min _{i} \min _{k} \Delta_{i}(k) .
\end{gathered}
$$

According to equations (12) and (13), it is necessary to find the extreme difference value, wherein $\lambda_{0}(M, m)$ corresponding to the first group $\Delta_{0}$ is the maximum value and the minimum value of the corresponding sequence of the group, and the rest is denoted as $\lambda_{1}, \lambda_{2}, \ldots, \lambda_{10}$; then, there are

$$
\begin{aligned}
& \lambda_{1}=(0.23,0), \\
& \lambda_{2}=(0.12,0), \\
& \lambda_{3}=(0.18,0), \\
& \lambda_{4}=(0.25,0), \\
& \lambda_{5}=(0.17,0), \\
& \lambda_{6}=(0.14,0), \\
& \lambda_{7}=(0.15,0), \\
& \lambda_{8}=(0.13,0), \\
& \lambda_{9}=(0.22,0), \\
& \lambda_{10}=(0.25,0) .
\end{aligned}
$$

From each limit value, find the extreme difference between the maximum value and the minimum value:

$$
\begin{aligned}
M & =0.25, \\
m & =0 .
\end{aligned}
$$

\subsection{Correlation Coefficient Sequence and Relevance}

Definition 1. The system behavior sequence and the comparison data sequence are as shown in equation (3), defining the resolution coefficient $\delta=\left(\gamma_{09}, \gamma_{01}, \gamma_{010}, \gamma_{07}, \gamma_{03}, \gamma_{08}\right.$, $\left.\gamma_{05}, \gamma_{04}, \gamma_{06}, \gamma_{02}\right) \in(0,1)$; let

$$
\begin{aligned}
\gamma\left(x_{0}(k), x_{i}(k)\right) & =\frac{\min _{i} \min _{k}\left|x_{0}(k)-x_{i}(k)\right|+\zeta \max _{i} \max _{k}\left|x_{0}(k)-x_{i}(k)\right|}{\left|x_{0}(k)-x_{i}(k)\right|+\zeta \max _{i} \max _{k}\left|x_{0}(k)-x_{i}(k)\right|}, \\
\gamma\left(X_{0}, X_{i}\right) & =\frac{1}{n} \sum_{k=1}^{n} \gamma\left(x_{0}(k), x_{i}(k)\right) .
\end{aligned}
$$

The given real number $\gamma\left(x_{0}(k), x_{i}(k)\right)$ constitutes a sequence of gray correlation coefficients. If the coefficient $\gamma\left(X_{0}, X_{i}\right)$ satisfies the gray correlation four axioms, it is called the gray correlation degree of $X_{i}$ to $X_{0}$. According to formulas (12), (13), (16), and (17), the following operation is performed, and the formula of the correlation coefficient is rewritten as

$$
\begin{gathered}
\gamma_{0 i}(k)=\frac{m+\zeta M}{\Delta_{i}(k)+\zeta M}, \quad \zeta \in(0,1), k=1,2, \ldots, n ; \\
i=1,2, \ldots, m .
\end{gathered}
$$

According to formula (18), the gray correlation coefficient sequence and Deng's gray correlation degree are derived:

$$
\begin{aligned}
& \beta_{0 i}=\left\{\gamma_{0 i}(1), \gamma_{0 i}(2), \ldots, \gamma_{o i}(k), \ldots, \gamma_{0 i}(n)\right\}, \\
& \gamma_{0 i}=\frac{1}{n} \sum_{k=1}^{n} \gamma_{0 i}(k) ; \quad i=1,2, \ldots, m .
\end{aligned}
$$

Since the weights of each sequence data are similar, the resolution coefficient $\zeta$ is 0.5 , and the abovementioned algorithm is implemented by $\mathrm{C}++$ program to obtain the corresponding data sequence: 


$$
\begin{aligned}
& \beta_{01}=\{0.42,0.68,0.49,0.86,0.61,0.42,0.53,0.81,0.53,0.71,1.00,0.35,1.00,0.61,0.61\}, \\
& \beta_{02}=\{0.86,0.81,1.00,0.58,0.53,0.86,0.93,0.51,0.86,0.64,1.00,0.64,0.81,0.76,0.64\}, \\
& \beta_{03}=\{0.41,1.00,1.00,0.86,0.56,0.41,0.58,0.76,0.49,0.47,1.00,0.56,1.00,0.56,0.56\}, \\
& \beta_{04}=\{0.45,1.00,0.56,0.42,0.86,0.40,0.47,1.00,0.93,0.42,0.81,0.33,0.49,0.58,0.93\}, \\
& \beta_{05}=\{0.42,0.51,0.81,0.64,0.47,0.86,0.93,0.58,0.64,0.61,1.00,0.71,1.00,0.86,0.61\}, \\
& \beta_{06}=\{0.68,1.00,0.81,0.47,0.68,0.76,0.47,0.64,0.49,0.76,0.64,0.56,1.00,0.81,0.81\}, \\
& \beta_{07}=\{0.45,0.68,0.68,0.68,0.45,0.81,0.68,0.68,0.81,0.58,1.00,0.58,1.00,0.81,0.81\}, \\
& \beta_{08}=\{0.56,1.00,0.81,0.56,0.81,0.56,0.56,0.81,0.68,0.68,0.56,0.68,1.00,0.49,0.68\}, \\
& \beta_{09}=\{0.53,1.00,0.36,0.86,0.47,0.86,0.53,1.00,0.86,0.47,0.68,0.86,0.36,0.71,0.86\}, \\
& \beta_{10}=\{0.38,0.81,1.00,0.61,0.71,0.38,0.71,0.33,0.47,0.76,1.00,0.53,1.00,0.42,0.53\} .
\end{aligned}
$$

The gray correlation degrees corresponding to $X_{0}$ are

$$
\begin{aligned}
& \gamma_{01}=0.642, \\
& \gamma_{02}=0.762, \\
& \gamma_{03}=0.681, \\
& \gamma_{04}=0.643, \\
& \gamma_{05}=0.710, \\
& \gamma_{06}=0.705, \\
& \gamma_{07}=0.713, \\
& \gamma_{08}=0.696, \\
& \gamma_{09}=0.694, \\
& \gamma_{10}=0.643 .
\end{aligned}
$$

In summary, $\gamma_{02}$ has the greatest correlation with the behavioral data sequence $X_{0}$ in the data sequence of 10 sets of virtual prototype performance tests, that is, the test index of the test cabin No. 2 is the most consistent with the assessment criteria.

In order to visually explain the accuracy of the gray correlation analysis, 10 sets of data are, respectively, made into data line diagrams composed of 10 sets of data sequences, as shown in Figure 2.

Figure 2 shows that the 15 performance indicators extracted from the cabin reliability analysis correspond to 15 coordinate points, respectively. According to the Deng's gray correlation analysis model, the closeness of the data lines $X_{1}, X_{2}, \ldots, X_{10}$ and $X_{0}$ is compared by 15 points. The value is judged by the proximity of the standard value (the corresponding value of the behavioral feature sequence). Therefore, Deng's gray correlation method is also called the "point correlation method" [9]. Through theoretical calculations, the gray relational order is obtained:

$$
\delta=\left(\gamma_{02}, \gamma_{07}, \gamma_{05}, \gamma_{06}, \gamma_{08}, \gamma_{09}, \gamma_{03}, \gamma_{04}, \gamma_{10}, \gamma_{01}\right)
$$

In Figure 2, the correlation between the corresponding 10 sets of comparison data sequence lines and the standard characteristic data sequence lines is consistent with the "point correlation method" analysis trend. According to the gray correlation sequence, the case of the virtual prototype cabins and standard characteristic data sequences of No. 2 and No. 7 is investigated.

As shown in Figure 3(a), it can be seen from the comparison between the data display and the test sample cabin No. 1 in Figure 3(b) that although $X_{02}$ has the greatest correlation with $X_{0}, X_{02}$ is not the best in shape similar to $X_{0} . X_{0}$ set of data line graphs, such as $X_{07}$, has a better degree of similarity. The so-called similarity is the degree to which the graph is consistent with the shape of the standard line graph E. Since the cabin has an economic cost as one of its design requirements, the similarity and proximity of the standard line should be considered separately. Obviously, C is more consistent with $\mathrm{B}$ in the similar aspect, but the point correlation does not clearly distinguish this. From the perspective of relevance, as shown in Figure 4, the higher the value, the better the relevance of the indicator.

In the figure, the 15-point correlation index between $\beta_{02}$ and $\beta_{07}$ is distributed between 0.4 and 1.0. If 8 is taken as the baseline, the virtual prototype of the 7 th has 6 indicators that meet the requirements, while the virtual prototype 2 has eight, and if 0.9 is used as the baseline, there are 2 on the 7 th and 3 indicators on the 2 nd, so the performance of the No. 7 virtual prototype is slightly worse.

The abovementioned results are consistent with Deng's related analysis results. The main reason is that Deng's gray correlation analysis model focuses on the similarity of the two curves on the relative displacement of several points on the curve, and the specific analysis does not reflect the characteristics of the system. The relative change rate of the curve and the comparison curve, that is, formula (16), contains only local parameters that reflect the closeness of the two curves and lacks the overall parameters that reflect the closeness of the two curves. The method is judged by the closeness of the limited point, and it cannot fully reflect the specific gray-related requirements such as economic factors, product demand factors, and social factors of the entire mechanical product research object. Therefore, a correlation analysis model based on a macroperspective is needed to ensure that the similarity of similar prototypes is not 
excluded. The introduction of generalized gray correlation analysis is to consider the relative rate of change factors. The meaning is here.

The biggest innovation in the generalized gray relational analysis system is that the similarity and proximity of the curves can be discussed separately for different engineering actual requirements.

\section{Generalized Grey Relational Analysis Model}

4.1. Generalized Grey Relational Method. The generalized gray relational analysis model has three objectives for the analysis of pod reliability: first, the curve approach of Deng's gray correlation analysis further demonstrates the basis of reliability optimal solution selection from a new perspective; second, the data sequence curve. The relative change rate problem between the two is added to the gray correlation analysis, and the original "point correlation analysis" is changed to the "face correlation analysis" between the curves. Starting from the overall situation of the curve distribution, the two curves are obtained by the starting point of zeroing. They are all placed on the coordinate axis, and the size of the area sandwiched between them is used as the criterion for judging the optimal solution of reliability analysis from the macroscopic perspective of the data. Third, the difference between the proximity and similarity of the curve is analyzed accordingly. Also, in the engineering practice, the corresponding factors of economic and design requirements are added. The generalized gray relational analysis is divided into three categories: absolute association method, relative association method, and comprehensive association method, which do not strictly satisfy the gray association four axioms.

\subsection{Grey Absolute Correlation Method}

Theorem 2. Set the behavior sequence $X_{i}=\left(x_{i}(1)\right.$, $\left.x_{i}(2), \ldots, x_{i}(n)\right)$ and record the corresponding polyline $X_{i}-$ $x_{i}(1)$ as $X_{i}-x_{i}(1)=\left\{x_{i}(1)-x_{i}(1), x_{i}(2)-x_{i}(1), \ldots, x_{i}(n)\right.$ $\left.-x_{i}(1)\right\}$; then,

$$
s_{i}=\int_{1}^{n}\left(X_{i}-x_{i}(1)\right) \mathrm{d} t
$$

Definition 2. Let the behavior sequence $X_{i}=\left(x_{i}(1)\right.$, $\left.x_{i}(2), \ldots, x_{i}(n)\right)$ and $D$ be defined as sequence operators and have the following relationship:

$$
\begin{aligned}
X_{i} D & =\left(x_{i}(1) d, x_{i}(2) d, \ldots, x_{i}(n) d\right), \\
x_{i}(k) d & =x_{i}(k)=x_{i}(1), \quad k=1,2, \ldots, n .
\end{aligned}
$$

According to equations (24) and (25), $D$ is called the starting point zeroing operator, and the starting point of $X_{i} D$ is zeroed, which is recorded as

$$
X_{i}^{0}=\left(x_{i}^{0}(1), x_{i}^{0}(2), \ldots, x_{i}^{0}(n)\right) .
$$

Theorem 3. We arbitrarily take two sequences of behavior:

$$
\begin{aligned}
& X_{i}=\left(x_{i}(1), x_{i}(2), \ldots, x_{i}(n)\right), \\
& X_{j}=\left(x_{j}(1), x_{j}(2), \ldots, x_{j}(n)\right) .
\end{aligned}
$$

The starting point zero image is

$$
\begin{aligned}
& X_{i}^{0}=\left(x_{i}^{0}(1), x_{i}^{0}(2), \ldots, x_{i}^{0}(n)\right), \\
& X_{j}^{0}=\left(x_{j}^{0}(1), x_{j}^{0}(2), \ldots, x_{j}^{0}(n)\right) .
\end{aligned}
$$

It is referred to as

$$
s_{i}-s_{j}=\int_{1}^{n}\left(X_{i}^{0}-X_{j}^{0}\right) \mathrm{d} t .
$$

If $X_{i}^{0}$ is always above $X_{j}^{0}$, then $s_{i}-s_{j} \geq 0$; if $X_{i}^{0}$ is always below $X_{j}^{0}$, then $s_{i}-s_{j} \leq 0$; and if $X_{i}^{0}$ intersects with $X_{j}^{0}$, the $s_{i}-s_{j}$ sign is indefinite.

Definition 3. The sequence of $X_{i}^{0}$ : the sum of the time intervals between the observation data is the length of $X_{j}^{0}$. It should be pointed out that the number of observations in two sequences of equal length is not necessarily equal.

Theorem 4. Let sequence $X_{0}$ and $X_{i}$ be of the same length, $s_{0}$ and $s_{i}$ are as described in equation (15) of Theorem 2, and $s_{i}-s_{0}$ is as described in equation (19) of Theorem 2; then,

$$
\varepsilon_{0 i}=\frac{1+\left|s_{0}\right|+\left|s_{i}\right|}{1+\left|s_{0}\right|+\left|s_{i}\right|+\left|s_{0}-s_{i}\right|} .
$$

Equation (30) is the absolute degree of gray correlation between $X_{0}$ and $X_{i}$.

According to the satisfaction condition, for 11 sets of data sequences, since the time intervals between adjacent observation data are the same, $X_{0}$ and $X_{1}, X_{2}, \ldots, X_{10}$ are said to constitute an isochronous sequence.

Theorem 5. Let sequence $X_{0}$ and $X_{i}$ be the same length, and both are 1-time-distance sequences, with the corresponding relationship:

$$
\begin{aligned}
& X_{0}^{0}=\left(x_{0}^{0}(1), x_{0}^{0}(2), \ldots, x_{0}^{0}(n)\right), \\
& X_{i}^{0}=\left(x_{i}^{0}(1), x_{i}^{0}(2), \ldots, x_{i}^{0}(n)\right) .
\end{aligned}
$$

The abovementioned sequence is the starting point zeroing image of $X_{0}$ and $X_{i}$, respectively, and the following correspondence is given:

$$
\begin{gathered}
\left|s_{0}\right|=\left|\sum_{k=2}^{n-1} x_{0}^{0}(k)+\frac{1}{2} x_{0}^{0}(n)\right|, \\
\left|s_{i}\right|=\left|\sum_{k=2}^{n-1} x_{i}^{0}(k)+\frac{1}{2} x_{i}^{0}(n)\right|,
\end{gathered}
$$




$$
\left|s_{0}-s_{i}\right|=\left|\sum_{k=2}^{n-1}\left(x_{i}^{0}(k)-x_{0}^{0}(k)\right)+\frac{1}{2}\left(x_{i}^{0}(n)-x_{0}^{0}(n)\right)\right|
$$

From equations (32)-(34), the following absolute correlation algorithm is derived:

$$
\varepsilon_{0 i}=\frac{1+\left|\sum_{k=2}^{n-1} x_{0}^{0}(k)+(1 / 2) x_{0}^{0}(n)\right|+\left|\sum_{k=2}^{n-1} x_{i}^{0}(k)+(1 / 2) x_{i}^{0}(n)\right|}{1+\left|\sum_{k=2}^{n-1} x_{0}^{0}(k)+(1 / 2) x_{0}^{0}(n)\right|+\left|\sum_{k=2}^{n-1} x_{i}^{0}(k)+(1 / 2) x_{i}^{0}(n)\right|+\left|\sum_{k=2}^{n-1}\left(x_{i}^{0}(k)-x_{0}^{0}(k)\right)+(1 / 2)\left(x_{i}^{0}(n)-x_{0}^{0}(n)\right)\right|}
$$

According to the gray absolute relevance theory, the related algorithm is written based on the $\mathrm{C}++$ programming language, and the starting point zeroing process is performed according to the data sequence obtained by $X_{0}$ and $X_{1}, X_{2}, \ldots, X_{10}$ :

$$
\begin{aligned}
& X_{0}^{0}=(0.00,2.00,-1.00,1.00,0.00,0.00,1.00,-1.00,1.00,1.00,2.00,0.00,-1.00,0.00,1.00) \\
& X_{1}^{0}=(0.00,1.40,-1.20,0.60,-0.30,0.00,0.90,-1.50,0.90,0.70,1.60,0.20,-1.60,-0.30,0.80) \\
& X_{2}^{0}=(0.00,2.10,-1.20,1.40,0.30,0.00,1.10,-0.80,1.00,0.80,2.20,0.20,-1.10,-0.20,1.30) \\
& X_{3}^{0}=(0.00,2.20,-0.40,1.30,0.20,0.00,1.10,-0.30,1.00,1.70,2.20,0.20,-0.40,-0.20,1.60) \\
& X_{4}^{0}=(0.00,1.60,-1.20,1.10,-0.40,0.10,1.00,-1.60,0.60,1.10,1.50,0.30,-1.10,-0.20,0.60) \\
& X_{5}^{0}=(0.00,1.30,-1.60,0.80,-0.10,-0.50,0.60,-1.40,0.80,0.30,1.70,-0.40,-1.70,-0.50,0.30) \\
& X_{6}^{0}=(0.00,2.20,-0.70,0.80,0.00,0.30,0.80,-0.60,1.60,1.10,2.00,-0.10,-0.80,0.10,1.30) \\
& X_{7}^{0}=(0.00,1.50,-1.40,0.80,0.00,-0.40,0.80,-1.40,0.50,0.90,1.70,-0.20,-1.60,-0.40,0.70) \\
& X_{8}^{0}=(0.00,2.30,-0.60,1.00,0.20,0.60,1.00,-0.60,1.50,1.30,2.00,0.10,-0.70,-0.10,1.50) \\
& X_{9}^{0}=(0.00,1.80,-0.70,0.80,0.10,-0.40,1.10,-1.40,0.80,1.20,1.60,-0.40,-0.70,-0.20,0.80) \\
& X_{10}^{0}=(0.00,1.40,-1.70,0.70,-0.50,0.00,0.60,-0.90,0.90,0.30,1.50,-0.30,-1.70,-0.10,0.80)
\end{aligned}
$$

The corresponding absolute relevance is

$$
\begin{aligned}
& \varepsilon_{01}=0.691, \\
& \varepsilon_{02}=0.932, \\
& \varepsilon_{03}=0.803, \\
& \varepsilon_{04}=0.80, \\
& \varepsilon_{05}=0.588, \\
& \varepsilon_{06}=0.882, \\
& \varepsilon_{07}=0.638, \\
& \varepsilon_{08}=0.824, \\
& \varepsilon_{09}=0.875, \\
& \varepsilon_{010}=0.592 .
\end{aligned}
$$

According to the theory of gray absolute correlation analysis, the corresponding $\mathrm{C}++$ program is written to calculate the gray absolute correlation order, which is discharged from left to right according to the degree of relevance:

$$
\tau=\left\{\varepsilon_{02}, \varepsilon_{06}, \varepsilon_{09}, \varepsilon_{08}, \varepsilon_{03}, \varepsilon_{04}, \varepsilon_{01}, \varepsilon_{07}, \varepsilon_{010}, \varepsilon_{05}\right\} .
$$

It can be seen from the results of the absolute correlation degree that the results based on the "face correlation method" are numerically different from those based on the "point correlation method," mainly because the considerations are different because the corresponding curve of $X_{1}, X_{2}, \ldots, X_{10}$ and $X_{0}$ to the absolute degree of correlation reflects the similarity between the curves.

Specifically, to evaluate the reliability of the test prototype, as shown in Figures 5(a) and 5(b), the area between the data line $X_{2}$ and the data line $X_{0}$ in (a) is smaller to the data line $X_{6}$ in Figure 5(b). The area between the data lines $X_{0}$ is essentially reflecting the similarity between the curves. After the starting point is zeroed, the starting points are classified into the same point, which is more convincing for the comparison of similarities. With reference to the design requirements of the pod cabin, the optimal solution of the pod structure is studied. The proximity of the standard index will be used as an important reference. In order to meet the requirements of high-quality structural design, No. 2 cabin should be selected in the sixth cabin.

4.3. Grey Relative Correlation Method. The gray relative association method is to characterize the approximate relationship between the behavior sequence $X_{0}$ and the rate of change of the comparison sequence $X_{i}$ relative to the starting point. The closer the rate of change of $X_{0}$ and $X_{i}$ is, the larger the relative correlation degree will be. 


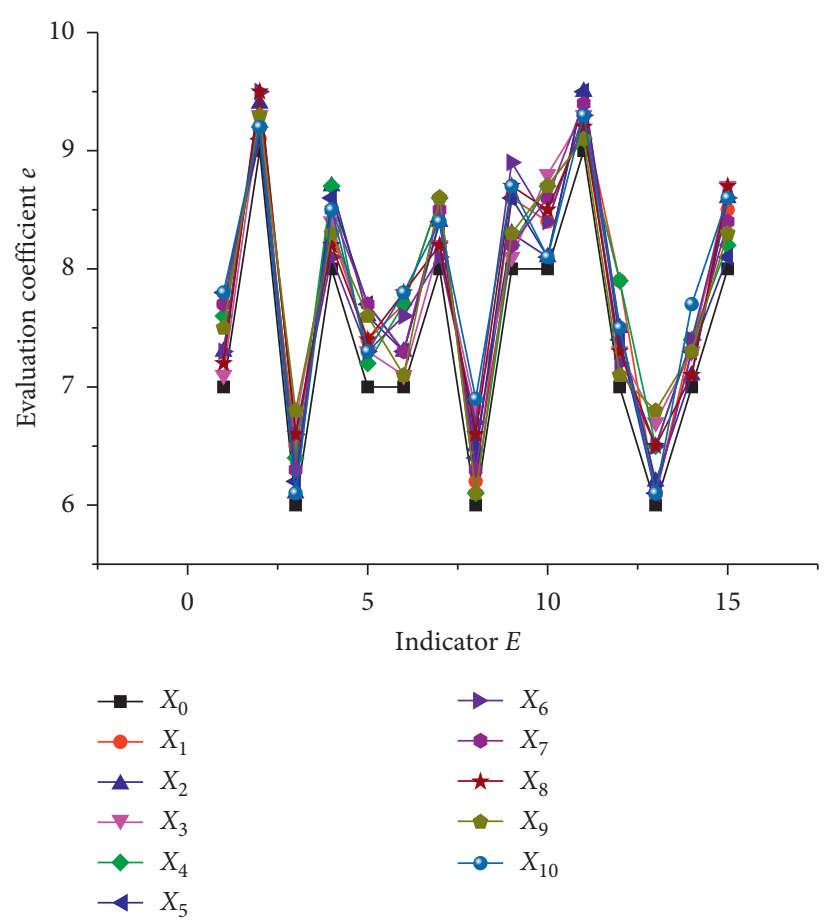

Figure 2: Data plot of the performance test of the pod cabin.

Definition 4. Let $X_{0}$ and $X_{i}$ have the same length, and the initial values are not equal to $0 . X_{0}^{\prime}$ and $X_{i}^{\prime}$ are their initial values, respectively, and then, the absolute correlation between $X_{0}^{\prime}$ and $X_{i}^{\prime}$ is the relative degree of $X_{0}$ and $X_{i}$.

According to the parameters of the existing data sequences $X_{0}$ and $X_{1}, X_{2}, \ldots, X_{10}$, the initial value processing is performed, that is, the data is divided by the first item number of the sequence, and the new series is obtained, which is consistent with the absolute gray correlation degree algorithm, and is written according to $\mathrm{C}++$. Program operation get its corresponding gray relative relevance:

$$
\begin{aligned}
& \gamma_{06}=0.930 \\
& \gamma_{07}=0.752, \\
& \gamma_{08}=0.874, \\
& \gamma_{09}=0.905, \\
& \gamma_{010}=0.698 \\
& \gamma_{01}=0.790 \\
& \gamma_{02}=0.961 \\
& \gamma_{03}=0.844 \\
& \gamma_{04}=0.853, \\
& \gamma_{05}=0.713
\end{aligned}
$$

It can be seen from the results of gray relative correlation that the change rate of the curve corresponding to $X_{2}$ is the closest to the relative starting point of $X_{0}$. The greater the relative correlation degree, the higher the approximation degree of the curve shape, which is reflected in the correlation degree value, that is, the relative test cabin No. $2 X_{0}$ has a high relative correlation. According to the gray relative association analysis theory, the gray relative correlation order is obtained, which is discharged from left to right according to the degree of relevance:

$$
\zeta=\left(\gamma_{02}, \gamma_{06}, \gamma_{09}, \gamma_{08}, \gamma_{04}, \gamma_{03}, \gamma_{01}, \gamma_{07}, \gamma_{05}, \gamma_{010}\right) \text {. }
$$

According to the parameters of the existing data sequences $X_{0}$ and $X_{2}, X_{6}$, the starting line is zeroed, and the graph is placed on the coordinate axis $x=0$, so that the correlation between the behavior data sequence line $X_{0}$ and the comparison data sequence line $X_{i}$ passes through two. The sequence is characterized by a rate of change from the starting point.

Specifically, the reliability of the test prototype was evaluated. As shown in Figures 5(a) and 5(b), relative to the starting point in (a), the rate of change of the data is better than the index in (b), which is essentially in the reflection curve. For the proximity between the two, refer to the design requirements of the pod and study an economical pod structure. It will be an important reference to the standard index of proximity. From the perspective of economy, choose No. 2 pod in cabin 6 .

4.4. Grey Comprehensive Association Method. The gray comprehensive correlation method combines the curve approximation considered by absolute correlation analysis with the similarity considered by relative correlation analysis, adds weight coefficient, and adjusts according to the 


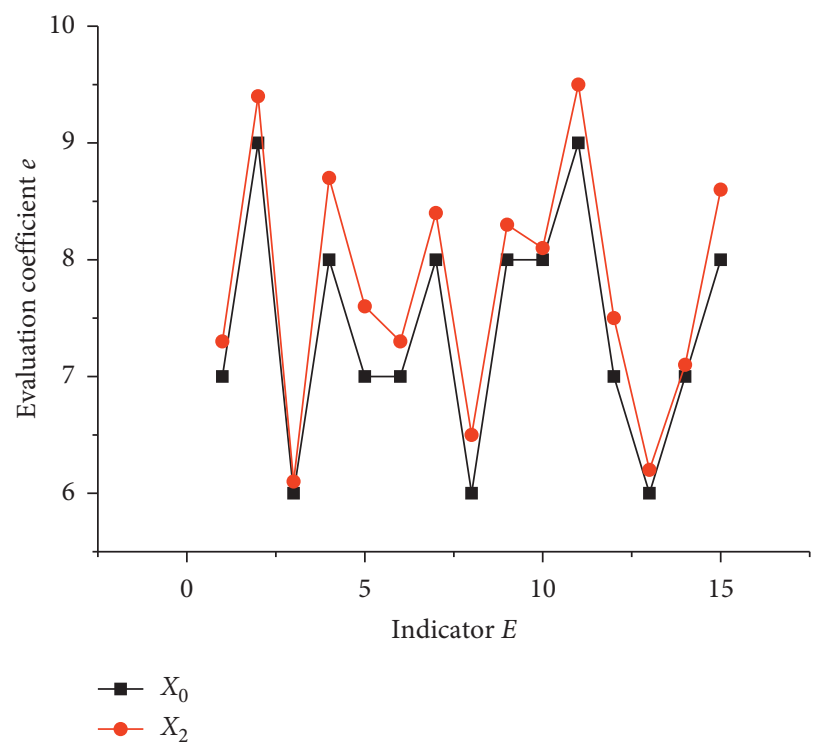

(a)

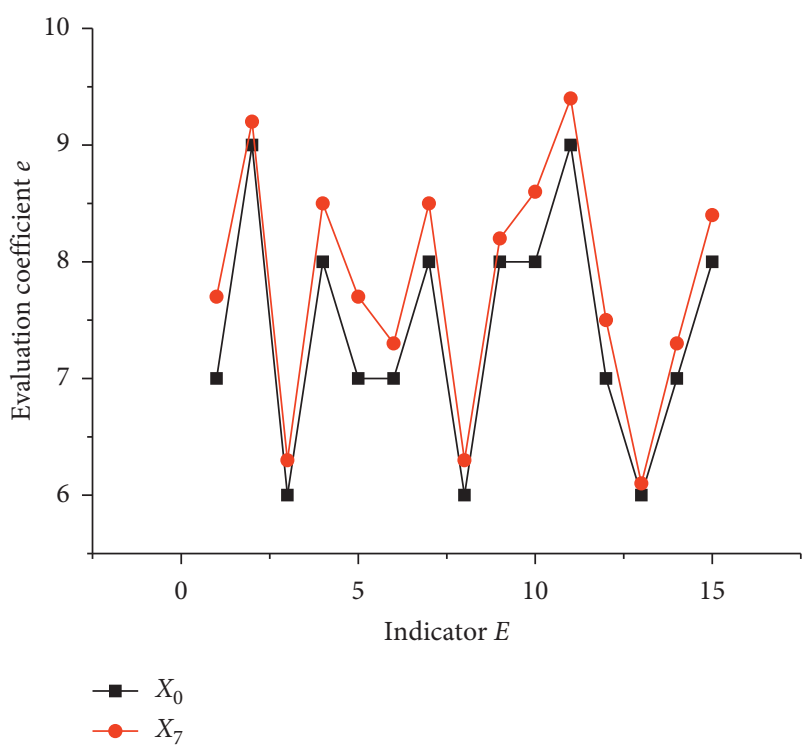

(b)

Figure 3: Data line diagram of the test cabin performance test for No. 2 and No. 7.

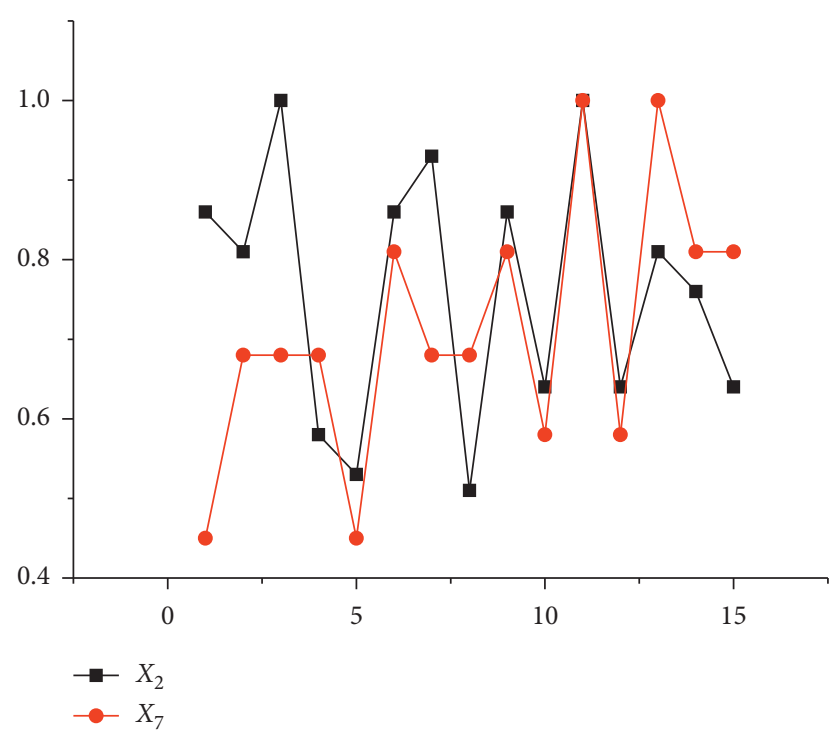

Figure 4: Analysis of the test performance of test cabins No. 2 and No. 7.

different emphasis of the technical parameters of airborne pod body to get the optimal data solution.

Definition 5. Let $X_{0}$ and $X_{i}$ have the same length, and the initial values are not equal to $0 . \varepsilon_{01}$ and $r_{01}$ are the absolute correlation and relative degree of $X_{0}$ and $X_{i}$, respectively, and $\theta \in(0,1)$ is called

$$
\rho_{0 i}=\theta \varepsilon_{0 i}+(1-\theta) r_{0 i},
$$

that is, the comprehensive degree of association between $X_{0}$ and $X_{i}$.

According to the abovementioned algorithm principle and the foregoing algorithm, the relative correlation operator is added on the basis of the absolute correlation degree, and the derived value is brought into the equation (41).

Due to technical requirements, the proximity and similarity of the reliability analysis curve of the pod cabin are important factors (taking economic factors as an important criterion, and the indicators far beyond the design standards will also be considered unreasonable); according to this reason, the weight coefficient is taken, after the operation, and the following data are obtained:

$$
\begin{aligned}
& \rho_{01}=0.741, \\
& \rho_{02}=0.947, \\
& \rho_{03}=0.824, \\
& \rho_{04}=0.827, \\
& \rho_{05}=0.651, \\
& \rho_{06}=0.906, \\
& \rho_{07}=0.695, \\
& \rho_{08}=0.850, \\
& \rho_{09}=0.890, \\
& \rho_{010}=0.645 .
\end{aligned}
$$

According to the comprehensive correlation analysis, it can be clearly seen that the test cabin No. 2 can meet the reliability evaluation index better than the test cabin of the sixth test cabin.

However, Deng's correlation analysis is the same as the abovementioned three correlation degree models. It is based on the proximity of the curve to infer the similarity between the curves. They still have a deficiency in distinguishing the two situations. In order to further distinguish the difference, a kind is given. The grey correlation degree is based on 


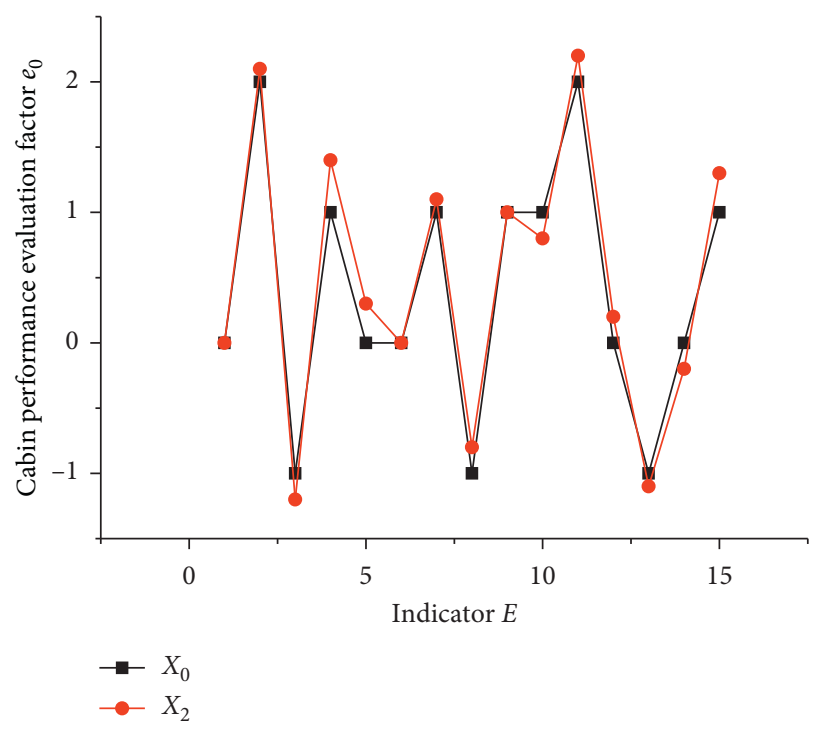

(a)

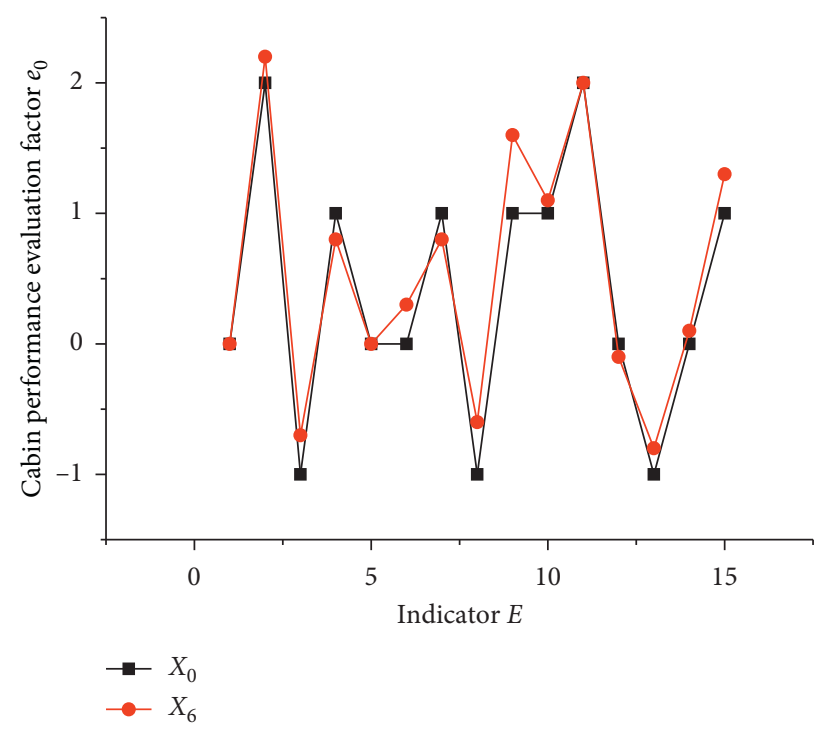

(b)

Figure 5: Test performance curve of test cabins No. 2 and No. 6 after zeroing at the starting point.

similarity and gray correlation degree model based on proximity [10].

\subsection{Similarity Gray Correlation Degree and the Proximity Gray Correlation Degree}

Definition 6. Let $X_{i}$ and $X_{j}$ be of the same length, $s_{i}-s_{j}$, as shown in equation (29); say

$$
\varepsilon_{i j}=\frac{1}{1+\left|s_{i}-s_{j}\right|} \text {. }
$$

It is the gray correlation degree between $X_{i}$ and $X_{j}$ based on similarity.

The similarity correlation is used to assess the degree of similarity between the sequences $X_{i}$ and $X_{j}$ in geometry. The more similar the $X_{i}$ and $X_{j}$ are in geometry, the larger the $\varepsilon_{09}$ value, and vice versa.

Definition 7. Let $X_{i}$ and $X_{j}$ be the same length, $s_{i}-s_{j}$, as shown in equation (27):

$$
s_{i}-s_{j}=\int_{1}^{n}\left(X_{i}-X_{j}\right) \mathrm{d} t .
$$

Then,

$$
\rho_{i j}=\frac{1}{1+\left|s_{i}-s_{j}\right|} .
$$

This is the proximity-based gray correlation between $X_{i}$ and $X_{j}$.

Proximity correlation is used to assess the proximity of sequence $X_{i}$ and $X_{j}$ in space. The closer the $X_{i}$ and $X_{j}$ are in space, the larger the $\rho_{i j}$ value, and vice versa. The similarity correlation only applies to sequences $X_{i}$ and $X_{j}$. The situation is exactly the same, which is the reason for the quality grading of the 15 performance indicators of the cabin frame.

Based on the abovementioned theory, two correlation analysis are performed on 10 sets of data sequences, and the results are shown in Table 2 .

According to Table 2, it can be seen that when the quality classification of the product of the pod cabin frame is carried out, the similarity gray correlation degree is used as the evaluation principle, that is, the overall geometric shape of the curve is similar and the "parallelism" is maintained within a certain range. Therefore, it is No. 2, No. 9, No. 6, No. 4, No. 8, No. 1, No. 3, No. 7, No. 10, and No. 5, when a certain characteristic of the product is analyzed as described in this section. For the proximity to be focused, the choice is $2,5,9$, $7,7,3,6,6,8,1$, and 10 .

It should be noted that the value of proximity is small, but it does not mean that the reliability of the product is low. For example, the corresponding line of No. 6 test prototypes is far higher than the standard line and far away from the standard thread, so the proximity is low, which fully meets the design requirements, but the economy is poor. In addition, all performance test indicators below the technical requirements in the project are unqualified, according to the trend of typical line diagrams shown in Figure 6:

According to Figures 3(b) and 6(a)-6(d), test capsules No. 4, No. 6, No. 7, No. 9, and No. 10 perform well in terms of proximity and similarity. However, as a design standard prototype, it is obviously unqualified. It is found that some of its evaluation points are lower than the standard index points (characteristic behavior sequence data values), so the scope of consideration is excluded. Referring to the curve of the test chamber No. 2 shown in Figure 3(a), the test cabin No. 2 of the comprehensive correlation degree $\rho_{02}=0.947$ is selected as the optima $l$ cabin to be assembled with the pod bracket and its components. 
TABLE 2: Cabin data curve correlation degree property evaluation ranking.

\begin{tabular}{|c|c|c|c|c|c|}
\hline Ranking & Symbol & Similarity & Ranking & Symbol & Proximity \\
\hline 1 & $\varepsilon_{02}$ & 0.5128 & 1 & $\rho_{02}$ & 0.1786 \\
\hline 2 & $\varepsilon_{09}$ & 0.4000 & 2 & $\rho_{05}$ & 0.1786 \\
\hline 3 & $\varepsilon_{06}$ & 0.3509 & 3 & $\rho_{09}$ & 0.1695 \\
\hline 4 & $\varepsilon_{04}$ & 0.2941 & 4 & $\rho_{07}$ & 0.1667 \\
\hline 5 & $\varepsilon_{08}$ & 0.2353 & 5 & $\rho_{03}$ & 0.1640 \\
\hline 6 & $\varepsilon_{01}$ & 0.2128 & 6 & $\rho_{04}$ & 0.1539 \\
\hline 7 & $\varepsilon_{03}$ & 0.2041 & 7 & $\rho_{06}$ & 0.1538 \\
\hline 8 & $\varepsilon_{07}$ & 0.1870 & 8 & $\rho_{08}$ & 0.1538 \\
\hline 9 & $\varepsilon_{010}$ & 0.1695 & 9 & $\rho_{01}$ & 0.1493 \\
\hline 10 & $\varepsilon_{05}$ & 0.1681 & 10 & $\rho_{010}$ & 0.1429 \\
\hline
\end{tabular}

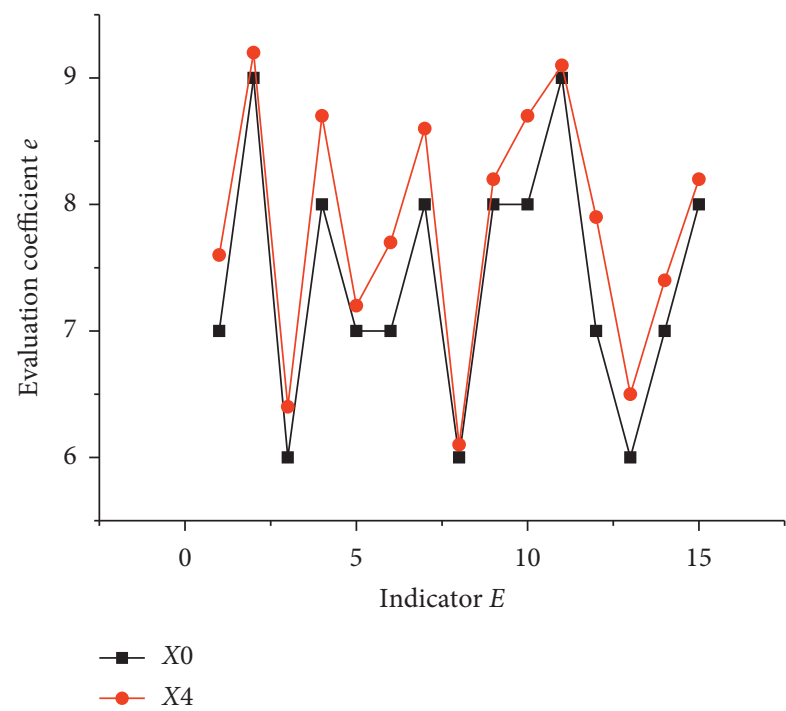

(a)

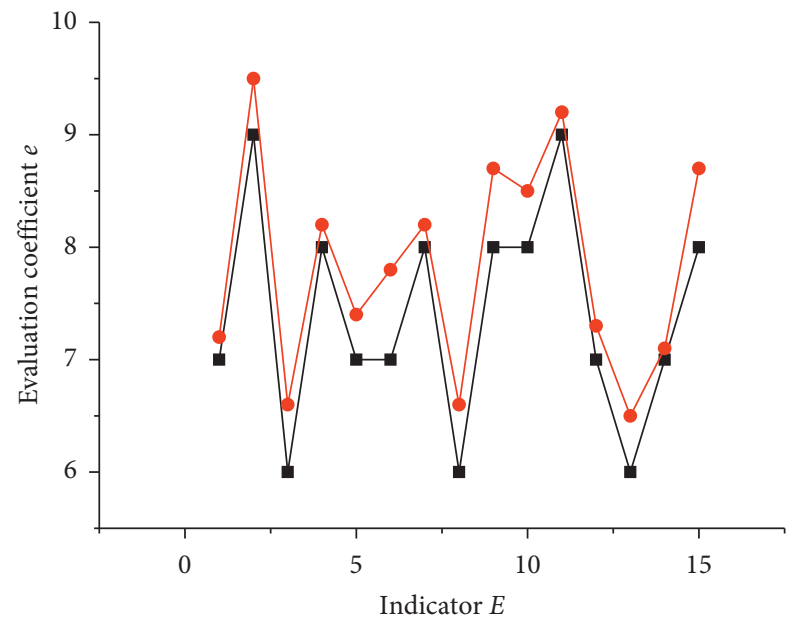

$$
\begin{aligned}
& \bullet X_{0} \\
& \rightarrow X_{8}
\end{aligned}
$$

(c)

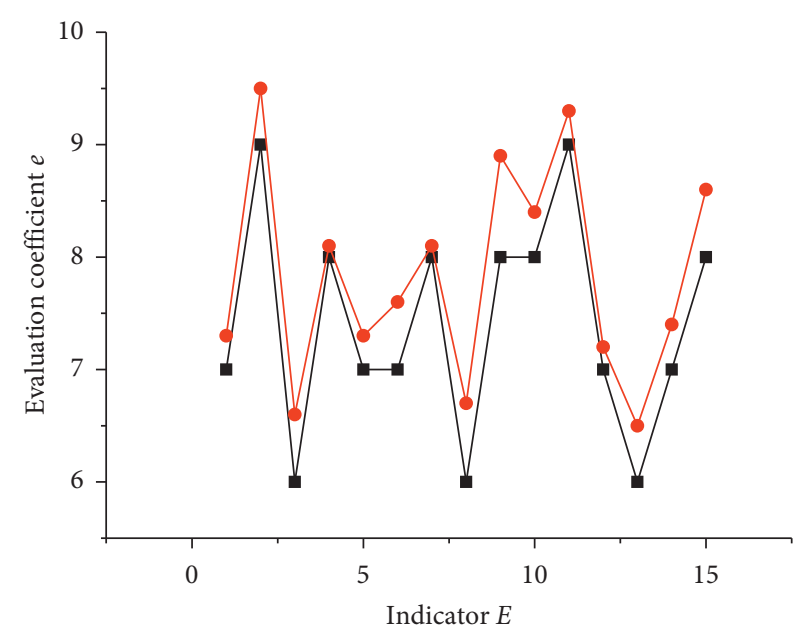

$\rightarrow X_{0}$

$\bullet X_{6}$

(b)

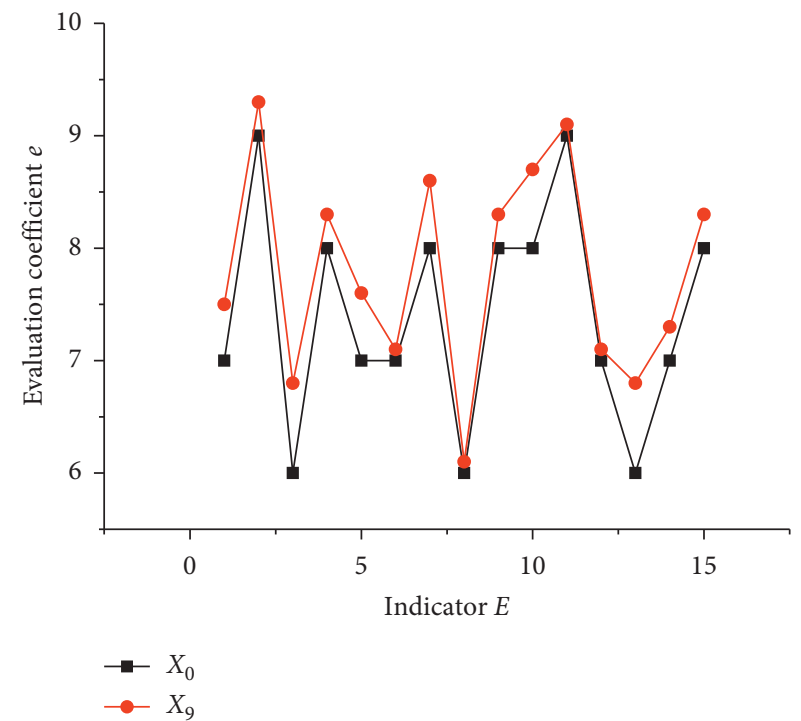

(d)

Figure 6: Performance test curve of test cabins No. 4, No. 6, No. 8, and No. 9. (a) Data line 4. (b) Data line 6. (c) Data line 8. (d) Data line diagram 9. 


\section{Conclusions}

(1) According to the gray system theory, designers can quickly and effectively optimize the less relevant cabins and propose improvements. It provides a numerical basis for further research on pod structure optimization and vibration safety inspection.

(2) Both Dun's gray correlation method and the generalized gray correlation method select the test cabin No. 2 as the optimal solution. However, it can be found that the possibility that the similar test chamber No. 6 is selected as the optimal solution still exists, for the "point correlation." The conclusions are often more convincing with the analysis of "face associations," and the considerations are more comprehensive and thorough.

(3) In the generalized gray relational analysis model, the concept of relative change rate between data curves is introduced. Starting from the overall situation of curve distribution, the size of the area between them is used as the criterion for judging the selection of the optimal test prototype for such aviation products, the basis.

(4) In the virtual design of the airborne pod, the reliability analysis of the pod cabin based on the generalized correlation method is introduced. The data curve similarity and proximity are practically applied in the reliability analysis of the pod structure, and the details are as follows. For the difference and connection of the reliability evaluation of mechanical products, the optimal solution for the economic and design requirements is obtained as the No. 2 cabin.

(5) Through the analysis of the generalized correlation method for each evaluation index by the relative gray correlation model, the reliability analysis of the airborne pod cabin frame is finally realized, which provides a specific theoretical basis for determining the optimal structure of the airborne pod cabin frame.

\section{Data Availability}

No data were used to support this study.

\section{Conflicts of Interest}

The authors declare that they have no conflicts of interest.

\section{Acknowledgments}

The authors gratefully acknowledge the National Nature Science Foundation (Project no. 51775172) and the Key Scientific and Technological Project of Luoyang City, Henan Province (no. 1801006A) for providing financial support for this research.

\section{References}

[1] N. Xie, W. Dong, and R. Mierzwiak, "Classification of research problems in grey system theory based on grey space concept," The Journal of Grey System, vol. 31, no. 1, pp. 100-111, 2019.

[2] W. Feng, J. Zhang, and X. Fu, "Grey system theory and its application in mechanical engineering," China Metal Bulletin, no. 10, p. 144, 2018.

[3] G. Sun, Research on Reliability Analysis on Key Components of Rotary Drilling Rig, Tianjin University, Tianjin, China, 2012.

[4] Y. Wang, "Reliability analysis of ship mechanical equipment based on stress-strength interference model," Ship Electronic Engineering, vol. 39, no. 7, pp. 139-141, 2019.

[5] Z. Wang, L. Qiang, Y. Hu, and Q. Wang, "Novel method for predicting SPE flux levels based on GM $(1,1)$ model and grey interval predictions," Journal of Beijing University of Aeaonautics and Astronautics, vol. 40, no. 8, pp. 1134-1142, 2014.

[6] Z. Wang, Q. Wang, and J. Fu, "Pressure multi-sensor data fusion and estimation of poor information based on bootstrap-fuzzy method," Journal of Beijing University of Aeronautics and Astronautics, vol. 39, no. 11, pp. 1426-1430, 2013.

[7] X. Liu, M. Gong, Z. Zhou, B. Li, and J. Dong, "An efficient mechanical structural reliability analysis method based on evidence theory," China Mechanical Engineering, pp. 1-9, 2020.

[8] S. Liu, Grey System Theory and Its Application, Science Press, Beijing, China, 2014.

[9] H. Yue and S. Liu, "Study on the characteristics of the development application and diffusion of grey system theory," Journal of Systems Science, vol. 16, no. 4, pp. 14-21, 2008.

[10] H. Wang and Y. Wei, "Axiomatization of the relevance degree of panel data based on proximity and similarity," Statistics \& Decision, vol. 35, no. 3, pp. 13-16, 2019. 